\title{
The analysis for heat island effect changes in Guiyang City base on remote sensing
}

\author{
Zhengtong Yin, Yongkang Zheng \\ College of Resources and Environmental Engineering, Guizhou University, Guiyang, China, 550025 \\ Email address: \\ yinzt33@gmail.com (Zhengtong Yin), 463924303@qq.com (Yongkang Zheng)
}

To cite this article:

Zhengtong Yin, Yongkang Zheng. The Analysis for Heat Island Effect Changes in Guiyang City Base on Remote Sensing. International Journal of Environmental Monitoring and Analysis. Vol. 2, No. 6, 2014, pp. 361-365. doi: 10.11648/j.ijema.20140206.20

\begin{abstract}
We selected the Landsat ETM+ remote sensing images of Guiyang city in 2003、2007 and 2011. These images was classified into land use charts and land surface temperature charts using ENVI software, found the changes of heat island effect of Guiyang City in this 8 years, then analyzed the relationship between land surface temperature and land use, The result showed that with the development of city, the heat island effect aggravated, almost all of the lower temperature regions transfer to high grade temperature zone, the low temperature region decline sharply, the medium temperature region has increased, high and higher temperature region increased largely.
\end{abstract}

Keywords: Remote Sensing, Heat Island, Land Surface Temperature Retrieval, Land Use Classification

\section{Introduce}

Due to population and economic growth, the population continues to concentrate in urban from rural and remote underdeveloped areas. Soared city buildings, expand the scale of the city as well as the consumption of resources has led to increasing anthropogenic heat rapidly, which makes the city the internal temperature is generally higher than rural and peri-urban areas. This heat island effect not only poses a serious impact on human life, and even a threat to human health. July 1980, St. Louis, and Kansas City, suffered a rare heat wave. In urban the business district heat island affected, its mortality rate rose by $57 \%$ and $64 \%$, while the suburban areas unaffected, the mortality rate did not rise by $10 \%$ [1].

Guiyang City, with its unique geographical advantages and climatic conditions won "a paradise under the Jervois, climate appropriate number of Guiyang" reputation. In view of the excellent weather conditions of Guiyang, the Guiyang City was awarded the "China summer capital "honor by the China Meteorological Society in 2007 [2]. However, research shows that extreme temperature days in Guiyang City increased [3], the heat island effect became intensified in recent years. In order to build our city well, we have done related research.

\section{Materials and Methods}

\subsection{Study Area}

Guiyang, capital of Guizhou province is Located in the eastern part of the Yunnan-Guizhou Plateau. Guiyang belong to mild humid subtropical climate. Due to the monsoon and topography, Guiyang City is cloudy and rainy all year, it is warm and suitable for human living. Its annual average temperature is $15.3{ }^{\circ} \mathrm{C}$. This paper studies the range of Guiyang City area, an area of 2381 square kilometers.

\subsection{Data Selection and Preprocessing}

Data used in this paper is Landsat $7 \mathrm{ETM}+$ satellite images, imaging time was September 26, 2003, September 21, 2007 and August 31,2011. Use the images which the track number is 127 / 41,127 / 42, we synthetic three cloudless images of study area. Because Landsat 7 satellite sensor was malfunctioning in 2003, its products edge had the phenomenon of missing bands, and Guiyang is located in the middle of the image area, fewer missing parts, so the pre-patch brings the error can be ignored.

Product data came from geospatial data cloud provider. It was dealt with image geometric correction via Envi4.7 TM remote sensing image processing software, and the accuracy was within 0.7 pixel. Then it was done atmospheric correction in scaled and visible band. The two images of the same phases 
at different track number was mosaicked, and then using Guiyang SAR image to crop mosaic map, we got three phases of Guiyang imagery.

\subsection{Surface Temperature Retrieval}

There are many surface temperature retrieval method based on remote sensing images, mainly single window algorithm, split window algorithm, the radiative transfer equation method, and various combinations of single-channel algorithms [4-5]. For Landsat 7 images, as it has only one thermal infrared channel (B62 band), so it does not need split window algorithm for multiple thermal infrared channels to eliminate the impact of high precision atmospheric radiation, the current mainstream approach is Zhihao Qin's single window algorithm, radiative transfer equation method and three kinds of universal single-channel algorithm [6-9]. In this article we choice radiative transfer equation which retrieval accuracy is better as Guiyang surface temperature retrieval.

Radiative transfer equation is also known as atmospheric correction method.

Expressions of thermal infrared radiance values which satellite sensors received can be written as (radiative transfer equation):

$$
\mathrm{L} \lambda=[\varepsilon \cdot \mathrm{B}(\mathrm{Ts})+(1-\varepsilon) \mathrm{L} \downarrow] \cdot \tau+\mathrm{L} \uparrow
$$

$\mathrm{L} \downarrow$ and $\mathrm{L} \uparrow$ denote atmospheric radiation upstream and downstream radiation, $\tau$ is the atmospheric transmittance in the thermal infrared band. This three parameters can be estimated by using the atmospheric correction software 6S, LOWTRAM or MODTRAN based on transmission equation preparation. $\mathrm{L} \lambda$ is the thermal infrared brightness sensor reception, $\varepsilon$ is the surface emissivity, TS is the true surface temperature, $\mathrm{B}(\mathrm{TS})$ is derived to the black body heat radiance in the TS through Planck's law. Through conversion formula (1), the blackbody with temperature $\mathrm{T}$ its radiation luminance $\mathrm{B}$ (TS) in the thermal infrared band is as follow:

$$
\mathrm{B}(\mathrm{Ts})=[\mathrm{L} \lambda-\mathrm{L} \uparrow-\tau \cdot(1-\varepsilon) \mathrm{L} \downarrow] / \tau \cdot \varepsilon
$$

Reuse Planck radiation function to find Ts

$$
\mathrm{Ts}=\mathrm{K} 2 / \ln (\mathrm{K} 1 / \mathrm{B}(\mathrm{Ts})+1)
$$

For $\mathrm{ETM}+, \mathrm{K} 1=666.09 \mathrm{~W} /(\mathrm{m} 2 \cdot \mathrm{sr} \bullet \mu \mathrm{m}), \mathrm{K} 2=$ 1282.71K;

Surface compare to emissivity as an important parameter to temperature retrieval have been done many studies before [10-11], we use the methods of previous studies, and the image of study area is divided into water bodies, built-up area, and natural surfaces three types. Like water pixel emissivity assignment 0.995, natural surfaces and built-up area pixel emissivity formula estimates are as follows:

$$
\begin{gathered}
\varepsilon_{\text {surface }}=0.9625+0.0614 \mathrm{FV}-0.0461 \mathrm{FV} 2 \\
\varepsilon_{\text {building }}=0.9589+0.086 \mathrm{FV}-0.0671 \mathrm{FV} 2
\end{gathered}
$$

$\varepsilon_{\text {surface }}$ and $\varepsilon_{\text {building }}$ represent natural surface and built-up areas emissivity, FV is the vegetation coverage being calculated by unmixing method, the principle is the assumption that NDVI value of Pixel is composition of the spectral values reflected by the bare soil mixed spectral values reflected by the pure vegetation, by obtaining NDVIS, NDVIV finally get vegetation coverage. Calculated as follows:

$$
\mathrm{FV}=(\text { NDVI-NDVIS) } /(\text { NDVIV-NDVIS })
$$

FV represents vegetation coverage, NDVIV and NDVIS spectral values represent pure reflection of vegetation and bare soil, take NDVIV $=0.70$ and NDVIS $=0.00$. And when a pixel of NDVI greater than $0.70, \mathrm{FV}$ value of 1 ; when NDVI is less than $0.00, \mathrm{FV}$ is 0 . NDVI is the normalized difference vegetation index, calculated as follows:

$$
\mathrm{NDVI}=(\mathrm{NIR}-\mathrm{R}) /(\mathrm{NIR}+\mathrm{R})
$$

NIR and R represent near-infrared and red bands which are 4-band and 3-band in TM images.

Finally, temperature maps were inverted according to the following rules divided temperature zones.

Table 1. The rules of divided temperature zones.

\begin{tabular}{lll}
\hline NO & degree & scope \\
\hline 1 & Higher temperature & $\mathrm{T} \geq 35^{\circ} \mathrm{C}$ \\
2 & high temperature & $30^{\circ} \mathrm{C} \geq \mathrm{T}>35^{\circ} \mathrm{C}$ \\
3 & Medium temperature & $25^{\circ} \mathrm{C} \geq \mathrm{T}>30^{\circ} \mathrm{C}$ \\
4 & low temperature & $20^{\circ} \mathrm{C} \geq \mathrm{T}>25^{\circ} \mathrm{C}$ \\
5 & Lower temperature & $\mathrm{T} \leq 20^{\circ} \mathrm{C}$ \\
\hline
\end{tabular}

\subsection{Land Use Classification}

To further investigate the relationship between changes of heat island and land use types in Guiyang, the images of the three-phase were classified as type of land use. The study area is divided into rough vegetation, building area, arable land area and water area this four categories. The true color images combined different band into were taken as a base map, referencing similar vintage land use map and high resolution Google Earth images establish interpretation, by the calculation, the selected sample separation are more than 1.90. Using the maximum likelihood method for image classification, obtained land use classification map, and then selected a certain validation samples for confusion matrix analysis, calculated the overall classification accuracy of this three images. They were more than $90 \%$, kappa coefficients were also 0.89 or more.

\section{Results and Analysis}

\subsection{Changes in Surface Temperature}

Guiyang City surface temperature retrieval results (Figure 1, Figure 2, Figure 3) show: in September 26, 2003 in Guiyang, there were large areas of medium and low temperature zone, were only sporadic high, higher and lower temperature zone. In September 21, 2007 in Guiyang City, most areas is still in the medium and low temperature zone, but the higher and high temperature zone significantly expanded compared with 2003, 
mainly in urban. In August 30, 2011 in Guiyang, most areas were in the medium and high temperature zone, and expanded significantly compare to 2007. Lower, low and higher temperature zone were only sporadic presence. The temperature zones change can be seen from Table 2, gone with these eight years, the lower temperature zone disappeared, the low temperature zone was sharp decline, the medium temperature zone increased, high and higher temperature zone soared. This three surface temperature retrieval maps were dealt with matrix change analysis, results can be seen from Table 3 that from 2003 to 2007, the low zone transformed into the medium zone was the largest area, $250.77 \mathrm{Km} 2$, accounting for about $10 \%$ of Guiyang City area; the area of medium zone into high zone was followed, $184.56 \mathrm{Km} 2$; medium zone transformation for the low zone was also larger area, $175.49 \mathrm{Km} 2$; the area of other transformation was smaller. As can be seen from Table 4, from 2007 to 2011, area of low zone transformed into the medium zone was the largest, $787.22 \mathrm{Km} 2$, accounting for about $33 \%$ of Guiyang City area; followed by medium zone into high zone, an area of 540.97 $\mathrm{Km} 2$, accounting for about $23 \%$ of Guiyang City area; other converted area was smaller. The overall trend is low grade transformed into high grade temperature zone.

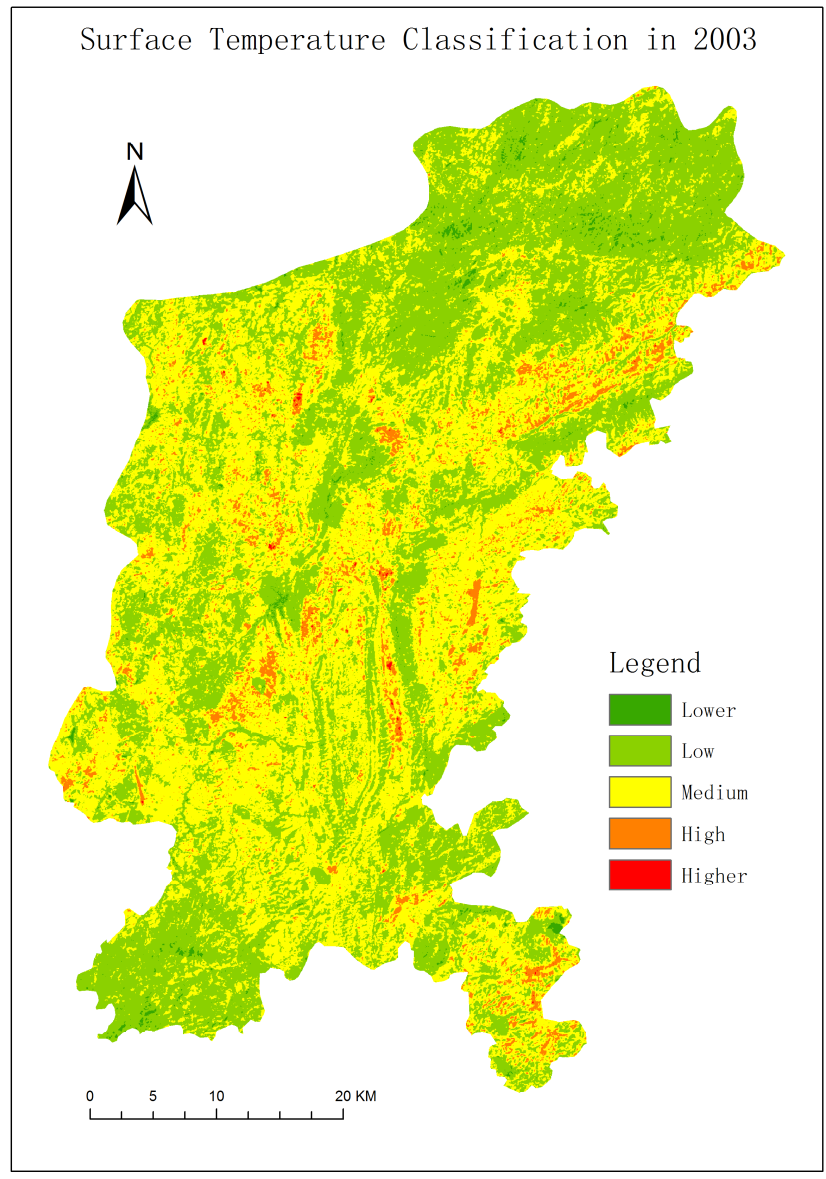

Figure 1. surface temperature in 2003.

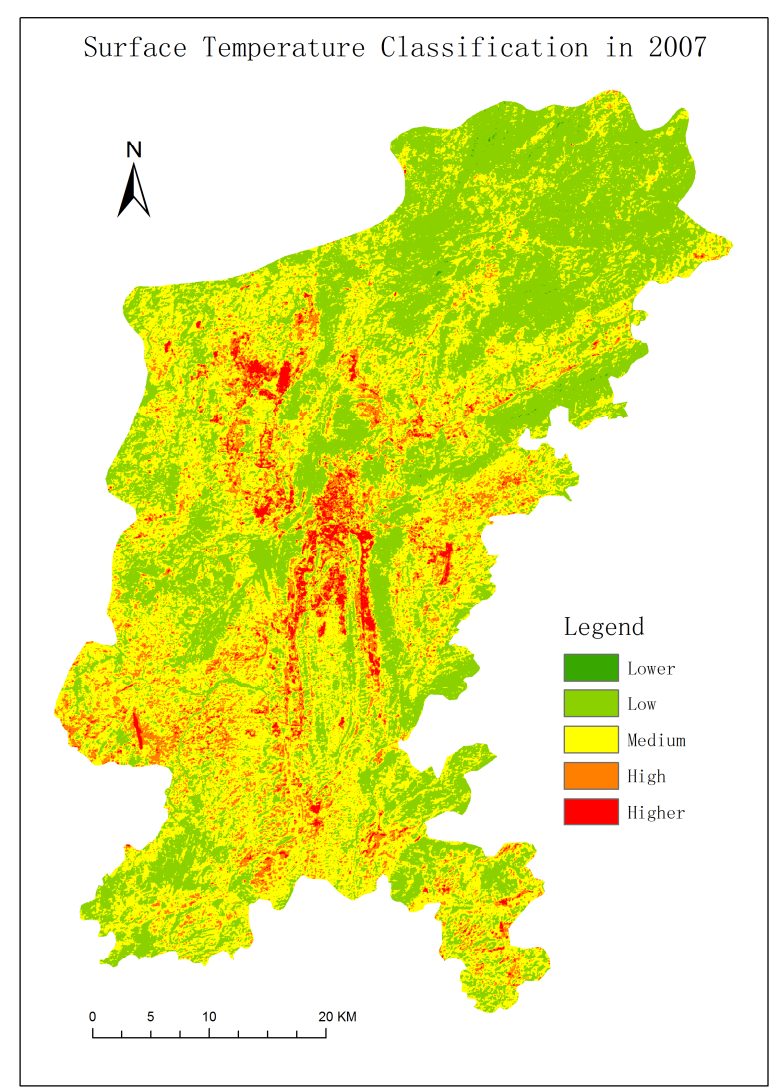

Figure 2. surface temperature in 2007.

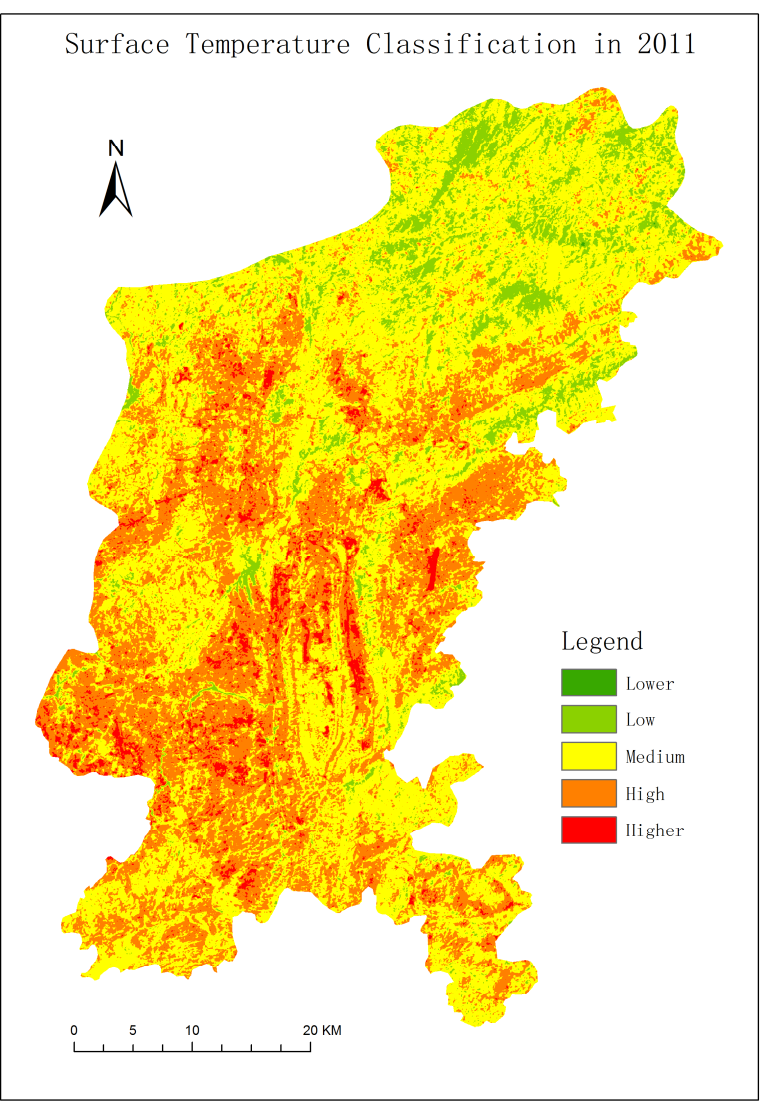

Figure 3. surface temperature in 2011. 


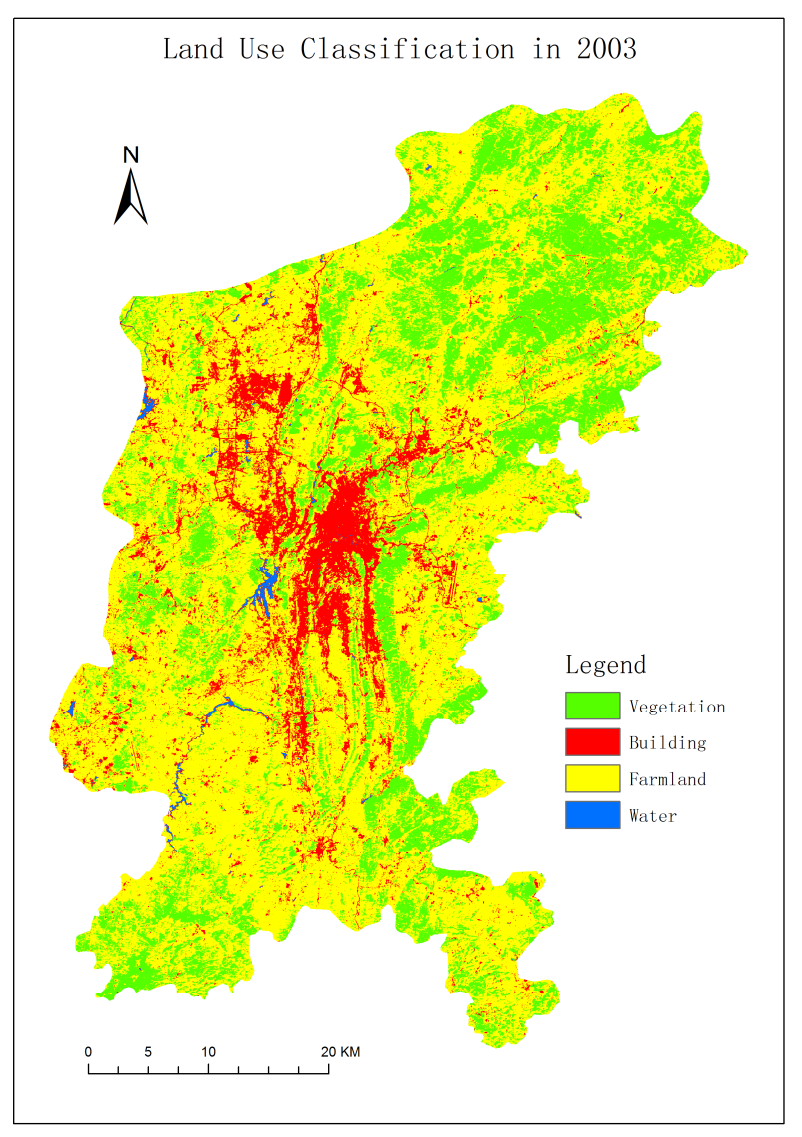

Figure 4. land use classification in 2003.

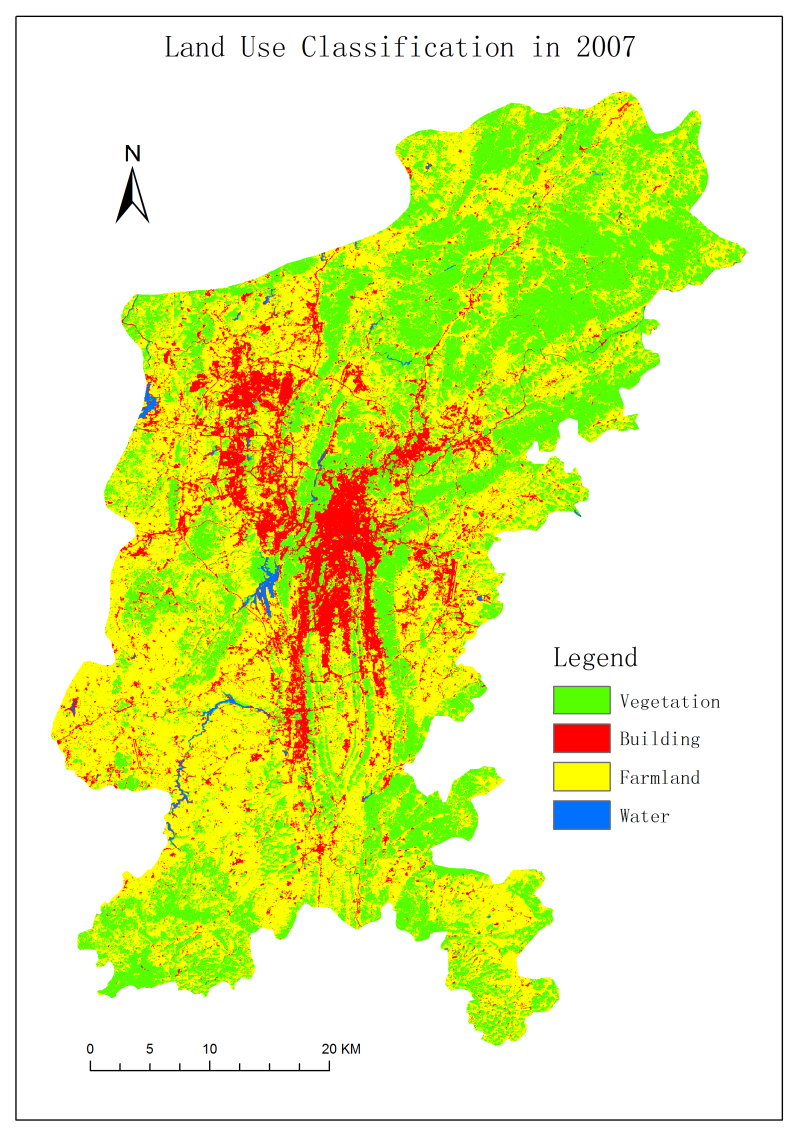

Figure 5. land use classification in 2007.

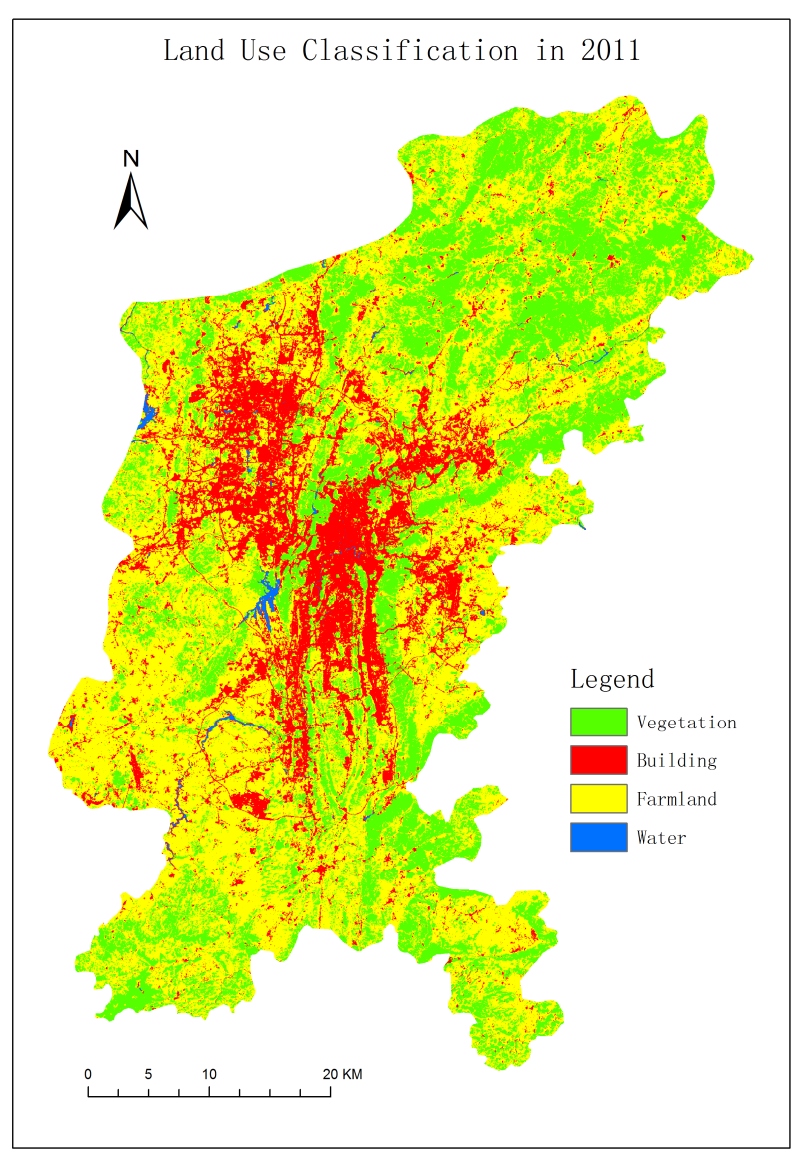

Figure 6. land use classification in 2011.

Table 2. The changes of each temperature zones $\mathrm{Km}^{2}$.

\begin{tabular}{lllllll}
\hline year & lower & low & medium & high & higher & total \\
\hline 2003 & 16.54 & 1091.50 & 1164.91 & 107.73 & 1.29 & \\
2007 & 1.03 & 1020.12 & 1067.36 & 236.58 & 56.68 & \\
2011 & 0.12 & 187.17 & 1302.45 & 814.42 & 77.65 & 2381.80 \\
\hline
\end{tabular}

Table 3. The transition matrix from 2003 to $2007 \mathrm{Km}^{2}$.

\begin{tabular}{lllllll}
\hline \multirow{2}{*}{2007} & $\mathbf{2 0 0 3}$ & & & & & \\
\cline { 2 - 7 } & lower & low & medium & high & higher & Total \\
\hline lower & 0.59 & 0.46 & 0.02 & 0.00 & 0.00 & 1.07 \\
low & 15.24 & 829.90 & 175.49 & 0.70 & 0.00 & 1021.33 \\
medium & 0.59 & 250.77 & 772.43 & 42.66 & 0.04 & 1066.49 \\
high & 0.10 & 9.49 & 184.56 & 42.15 & 0.12 & 236.42 \\
higher & 0.03 & 0.88 & 32.41 & 22.23 & 1.13 & 56.68 \\
Total & 16.54 & 1091.50 & 1164.91 & 107.73 & 1.29 & 2381.99 \\
\hline
\end{tabular}

Note: The table column "Total" says the 2007 data for each temperature zone area, row "Total" data indicates the 2003 data for each temperature zone area.

Table 4. The transition matrix from 2007 to $2011 \mathrm{Km}^{2}$.

\begin{tabular}{lllllll}
\hline \multirow{2}{*}{2011} & $\mathbf{2 0 0 7}$ & & & & & \\
\cline { 2 - 7 } & lower & low & medium & high & higher & Total \\
\hline lower & 0.00 & 0.11 & 0.01 & 0.00 & 0.00 & 0.12 \\
low & 0.99 & 183.40 & 2.77 & 0.03 & 0.00 & 187.20 \\
medium & 0.03 & 787.22 & 502.80 & 11.81 & 0.54 & 1302.41 \\
high & 0.01 & 47.39 & 540.97 & 193.97 & 32.06 & 814.40 \\
higher & 0.00 & 2.00 & 20.81 & 30.77 & 24.07 & 77.65 \\
Total & 1.03 & 1020.12 & 1067.36 & 236.58 & 56.67 & 2381.78 \\
\hline
\end{tabular}

Note: The table column "Total" says the 2011 data for each temperature zone area, row "Total" data indicates the 2007 data for each temperature zone area. 


\section{2. Analyzing the Relationship}

Comparing the land use classification (Figure 4, Figure 5, Figure 6, the year followed by the corresponding figure 1,2,3) with the temperature rating chart(figure 1,2,3), it is clear that significant regional of heat island concentrated in urban where building is density. Table 5 shows that Guiyang construction area grew rapidly in the past 11 years, in 2011 the construction area is 1.6 times in 2003. Corresponding growth of construction areas, temperature rate also enhanced rapidly, the heat island effect aggravated. The Yunyan District and Jinyang New District were the most obvious. On the other hand, in the vegetation area the temperature rate is lower or low and arable land area is mainly medium. This shows that the urban heat island and city building density have a very close relationship.it

Table 5. The changes of land use $\mathrm{Km}^{2}$.

\begin{tabular}{llllll}
\hline year & vegetation & construction & arable & water & Total \\
\hline 2003 & 623.09 & 238.86 & 1509.15 & 10.70 & \\
2007 & 876.03 & 289.24 & 1203.11 & 13.42 & \\
2011 & 746.40 & 386.94 & 1236.93 & 11.53 & 2381.80 \\
\hline
\end{tabular}

\section{Conclusions and Insufficient}

\subsection{Conclusion}

Compared to other heat island research methods, the advantages of remote sensing is rapid data capture and broad scope of the study. Using Landsat 7 ETM + images for the temperature retrieval and land use classification, we got more accurate results for Guiyang heat island changes. Studies show that:

1) 8 years 2003-2011, the lower temperature zone almost completely shifted to high-grade temperature zones, low temperature zone sharply declined, the medium temperature zone has increased, a high and higher temperature zone increased considerably.

2) The heat island changes has a very close relationship with changes of vegetation and arable land, also has close relationship with changes of urban buildings.

\subsection{Insufficient}

1) Using remote sensing images for the surface temperature retrieval, the image quality requirements are very strict, and in Guiyang summer is high humidity, clouds, fog, it produce a great impact on remote sensing imaging, therefore optional data is limited.

2) The transit time Landsat satellite over Guiyang is about
11:30, and for the day the highest temperature is generally around $14: 00$, so there is a certain gap between the retrieval from the real heat island.

\section{References}

[1] Peng Shaolin, Zhou Kai, Ye Youhua, etc. Urban Heat Island Effect Research Progress [J] Ecology and Environment, 2005, 14 (4): 574-579.

[2] Chen Gang. Guiyang Crowned "Summer Capital" Title [EB / OL]. http://gb.cri.cn/14714/2007/08/31/1965@1742498.htm [2014-09-15].

[3] Li Zhanjie, Yu Jingshan, Xin Da. Temperature Variation Analysis of Guiyang From 1951 to 2010 [J] South to North Water Transfers and Water Science \&Technology, 2011, 9 (5): 31-35.

[4] Mao Kebiao, Tang Huajun, Chen Zhongxin, etc. a Split Window Algorithm for retrieving Surface Temperature from ASTER data [J], Remote Sensing information, 2006, 5: 7-11.

[5] Wang Qianqian, Qin Zhihao, Wang Fei. A single Window Algorithm for retrieving Surface Temperature Based on Multi-source Remote Sensing Data [J] Geography and Geo-Information Science, 2012, 28 (003): 24-26.

[6] Qin Fuying. Based on Thermal Infrared Remote Sensing the Surface Temperature Retrieval Method Application and Comparative analysis Study [D]: Hohhot, Inner Mongolia Normal University, 2008.

[7] DING Feng, Xu Hanqiu. Comparative Analysis of Three Kinds of Surface Temperature Retrieval Algorithm Based on Landsat TM [J] Fujian Normal University: Natural Science, 2008 (1): 91-96.

[8] Qin Z, Karnieli A, Berliner P. A mono-window algorithm for retrieving land surface temperature from Landsat TM data and its application to the Israel-Egypt border region $[\mathrm{J}]$. International Journal of Remote Sensing, 2001, 22 (18): 3719-3746.

[9] Jiménez-Muñoz JC, Sobrino J A. A generalized single-channel method for retrieving land surface temperature from remote sensing data [J] Journal of Geophysical Research: Atmospheres (1984-2012), 2003, 108 (D22).

[10] Qin Zhihao, LI Wenjuan, Xu Bin, etc. the Surface Emissivity Parameters Estimation Method in Using Landsat TM6 for Surface Temperature Retrieval [J] Advances in Marine Science, 2005, 22 (B10):.. 129-137.

[11] Sobrino JA, Raissouni N, Li Z LA comparative study of land surface emissivity retrieval from NOAA data $[\mathrm{J}]$.Remote Sensing of Environment, 2001,75 (2): 256-266. 